\title{
Predominant conjugation with glycine of biliary and lumen bile acids in rats fed on pectin
}

\author{
BY T. IDE AND M. HORII \\ Laboratory of Nutrition Chemistry, National Food Research Institute, Ministry of \\ Agriculture and Fisheries, 2-1-2 Kannondai, Tsukuba Science City 305, Japan
}

(Received 1 June 1988 - Accepted 5 December 1988)

\begin{abstract}
1. Bile acids were analysed in the bile and lumen samples of rats which received a cholesterol-free or cholesterolenriched $(5 \mathrm{~g} / \mathrm{kg})$ diet free from fibre, or containing cellulose or citrus pectin at the level of $100 \mathrm{~g} / \mathrm{kg}$.

2. Dietary pectin but not cellulose increased biliary bile acid concentration and excretion. Dietary cholesterol did not affect biliary bile acids quantitatively.

3. Biliary bile acids were almost exclusively conjugated with glycine or taurine in the various experimental situations. The predominant portion of bile acids in rats fed on the cholesterol-free diet was conjugated with taurine when the diet was either free from fibre or contained cellulose; the ratio of bile acids conjugated with glycine: those conjugated with taurine $(G: T)$ was less than $0 \cdot 2$. In contrast, with pectin as a fibre source, the conjugation with glycine increased enormously $(\mathrm{G}:$ T increased to approximately 4). Cholesterol enrichment of the diet also increased the glycine conjugation in all groups of rats. Even in this situation, the $G: T$ was highest in rats fed on pectin.

4. Pectin, but not cellulose, increased the bile acid content of the small intestine and caecum, both in rats fed on the cholesterol-free and cholesterol-enriched diets. Cholesterol feeding doubled the bile acid content of the caecum in rats fed on a fibre-free diet or a cellulose diet, but not in those fed on pectin. No such effect of cholesterol was observed in the small intestine, except for the ileal bile acid content in rats fed on cellulose.

5. A considerable portion of the bile acids in the small intestine was deconjugated. The extent of the deconjugation was higher in the ileum than in the jejunum. As in the bile, G: T in rats fed on pectin (3.5.5) were higher than those in the other groups (0.05-1.05) in various situations. Also, cholesterol feeding considerably increased the ratio in all groups of rats.

6. The observed dietary alteration of the partition of bile acids between glycine and taurine may be of physiological significance in regulating bile acid and lipid metabolism in rats.
\end{abstract}

Bile acids are conjugated either with glycine or taurine in the liver by the action of the enzyme bile acid-CoA:amino acid $N$-acyltransferase before being excreted in the bile to the intestinal lumen (Jacobsen \& Smith, 1968; Killenberg, 1978; Elliott, 1985). Nonmammalian species conjugate bile acids exclusively with taurine, while mammalian species can synthesize both glycine- and taurine-conjugated bile acids. The ratio, glycine:taurine conjugates $(\mathrm{G}: \mathrm{T})$ varies considerably among mammalian species (Haslewood \& Wooton, 1950; Bremer, 1956; Jacobsen \& Smith, 1968; Elliott, 1985). Although conjugation with both glycine and taurine occurs in man, the glycine conjugates predominate under most conditions (Garbutt et al. 1971). In contrast, rats conjugate bile acids almost exclusively with taurine (Haslewood \& Wooton, 1950; Bremer, 1956; Jacobsen \& Smith, 1968; Killenberg, 1978; Elliott, 1985), and it has been thought that significant amounts of glycine conjugates were synthesized only under non-physiological conditions where intracellular taurine was limiting (Bergeret \& Chatagner, 1956; Daisy et al. 1956; Bengmark et al. 1964; Sturman, 1973; Hardison \& Proffitt, 1977). The substrate specificity of the hepatic $N$-acyltransferase (Killenberg \& Jordan, 1978) and active taurine synthesis (Jacobsen \& Smith, 1968) both accounted for the predominance of taurine conjugates in this animal species.

In human beings, $G: T$ is influenced by a variety of dietary, physiological and pathological factors (Lindstedt et al. 1965; Garbutt et al. 1971; Garbutt \& Kenney, 1972; Hardison, 1978; Elliott, 1985). Although the physiological signficance of this alternation is 
poorly understood, it may affect the overall processes of lipid absorption and metabolism. The existence of considerable differences in the various aspects of cholesterol metabolism among the mammalian species in which the G:T varies widely (Dietschy \& Wilson, 1970) may support this concept. As the rat has been considered to be an exclusive taurine conjugator, only a few studies (Bergeret \& Chatagner, 1956; Daisy et al. 1956; Yamanaka et al. 1985; Kawamura et al. 1986) have examined the influence of dietary factors on G:T in this animal species. However, growing evidence (Hardison, 1978; Yamanaka et al. 1985; Kawamura et al. 1986) indicates that the G:T in this animal species may be profoundly influenced by conditions where bile acid metabolism is modified. In the present study we have examined the effects of dietary fibre and cholesterol on this variable in rats.

\section{EXPERIMENTAL}

\section{Animals and diets}

Male rats of Wistar-Imamichi strain (4 weeks of age) were obtained from the Imamichi Institute of Animal Reproduction, Ibaraki. Animals were individually housed in a room with controlled temperature $\left(20-22^{\circ}\right)$, humidity $(55-65 \%)$ and lighting (lights on from 07.00 to 19.00 hours). All the animals were fed on a pelleted, commercial, non-purified diet (Type NMF; Oriental Yeast Co., Tokyo) and were acclimated to the housing conditions for at least $5 \mathrm{~d}$ after arrival. The rats were then randomly divided into groups of six to seven rats and assigned to cholesterol-free or cholesterol-enriched purified experimental diets, containing different types of dietary fibre or free from any fibre source. The basal composition of the purified diet was $(\mathrm{g} / \mathrm{kg})$ : casein 200 , maize oil 50 , maize starch 150 , mineral mixture 35 , vitamin mixture 10 , choline bitartrate 2 , sucrose to $1 \mathrm{~kg}$. Mineral and vitamin mixtures, in which the compositions were the same as those reported by the American Institute of Nutrition (1977), were obtaind from Nihon Nosan Kogyo K.K., Tokyo. Dietary fibre (cellulose or citrus pectin; $100 \mathrm{~g} / \mathrm{kg}$ ) and cholesterol $(5 \mathrm{~g} / \mathrm{kg}$ ) were added to the basal diet at the expense of sucrose. In the first experiment, rats were fed on the experimental diets for 26-28 d. A group of rats fed on a powdered, non-purified diet (Type NM; Oriental Yeast Co., Tokyo) was also included in the trial for comparative purposes.

Animals were anaesthetized with an intraperitoneal injection of Nembutal $(50 \mathrm{mg} / \mathrm{kg})$. The bile ducts were cannulated with a PE-10 tube and bile was allowed to drain for $2 \mathrm{~h}$ into a test-tube cooled on ice (Sugano et al. 1983). The rats were kept under anaesthesia with an additional injection of Nembutal $(25 \mathrm{mg} / \mathrm{kg}) 1 \mathrm{~h}$ after the initiation of biliary drainage. Faeces were collected for $2 \mathrm{~d}$ before the termination of the experimental period. In the second trial, rats were fed on the purified experimental diets for $28 \mathrm{~d}$. Animals were lightly anaesthetized with diethyl ether and blood was withdrawn from the inferior vena cava. The small intestine and caecum were rapidly removed and the small intestine was divided into two segments of equal length. The proximal and distal portions of the segments are referred to as jejunum and ileum respectively. Lumen contents were washed thoroughly with saline ( $9 \mathrm{~g}$ sodium chloride/l).

\section{Bile acid analyses}

The bile samples $(0.2 \mathrm{ml})$ were diluted with $3 \mathrm{ml} 0.5 \mathrm{M}$-phosphate buffer $(\mathrm{pH} 7.0)$ and passed through a commercial octadecylsilyl silica cartridge (Sep-Pak $\mathrm{C}_{18}$ ) which had been washed with $10 \mathrm{ml}$ each of chloroform, methanol and then water. The cartridge was washed with $10 \mathrm{ml}$ each of water and $1.5 \%$ ethanol, and bile acids were eluted with $5 \mathrm{ml} 90 \%$ ethanol (Goto et al. 1981). Lumen contents were homogenized with a polytron-type homogenizer. A portion of the homogenate $(5-10 \mathrm{ml})$ was mixed with $4 \mathrm{M}$-sodium hydroxide to give an alkaline concentration of $0.2 \mathrm{M}$. The mixture was heated at $60^{\circ}$ for $15 \mathrm{~min}$, centrifuged at 
$3000 \mathrm{rev} / \mathrm{min}$ for $10 \mathrm{~min}$ and the clear supernatant fraction was passed through a Sep-Pak $\mathrm{C}_{18}$ cartridge (Ide \& Horii, 1987). The cartridge was washed and the bile acids were recovered as described above. The extents of deconjugation of glycine- and taurineconjugated bile acids under the present conditions of alkaline treatment were found to be minimal (less than $0.2 \%$ ) (Ide \& Horii, 1987). The bile acids in the extract from bile and lumen contents were fractionated, according to the mode of conjugation, using a column of piperdinohydroxydextran gel (PHP gel; Shimazu Corp., Kyoto) as described elsewhere (Goto et al. 1981; Ide \& Horii, 1987). Faecal bile acids were extracted with ethanol under reflux (Uchida et al. 1977). The bile acids in these samples were determined by gas-liquid chromatography using AN-600 on gaschrom $\mathrm{Q}$ and nordeoxycholic acid as an internal standard (Kuriyama et al. 1979). The validity of the present method has been discussed elsewhere (Ide \& Horii, 1987). Faecal neutral steroids were also determined by gas-liquid chromatography, using $5 \alpha$-cholestane as an internal standard (Sugano et al. 1983).

\section{Lipid analyses}

Hepatic, serum and biliary lipids were extracted and purified (Folch et al. 1957). Triglyceride, cholesterol and phospholipid in the lipid extracts, except for biliary cholesterol, were chemically determined as described by Ide et al. (1978). Cholesterol in the bile sample was determined enzymically (Ide et al. 1982).

\section{Statistical analysis}

Values were analysed by a two-way analysis of variance and differences of means were inspected at $P<0.05$ (Snedecor \& Cochran, 1967).

\section{RESULTS}

\section{Effects of dietary fibre and cholesterol on biliary bile acids}

Biliary lipid compositions of rats fed on the non-purified diet and experimental purified diets are shown in Table 1. As the composition of the non-purified diet was completely different from those of the purified diets, statistical examination was made only among the groups of rats fed on purified diets. Among the rats assigned to the purified diets, both with and without cholesterol, the animals fed on pectin gained weight $(7 \cdot 1$ (SE 0.2) and 7.2 $(\mathrm{SE} 0 \cdot 2) \mathrm{g} / \mathrm{d}$ for animals fed on cholesterol-free and cholesterol-enriched diets respectively), slightly but significantly less than those fed on the diets free from any fibre source $(8 \cdot 2$ (SE 0.2$)$ and $8.5(\mathrm{SE} 0.4) \mathrm{g} / \mathrm{d}$ respectively), or those containing cellulose $(8.3(\mathrm{SE} 0.2)$ and 8.6 (SE 0.2$) \mathrm{g} / \mathrm{d}$ respectively). The retardation of growth in these animals can be ascribed to the reduced consumption of the diets $(18.3$ (SE 0.5 ) and 18.9 (SE 0.4 ) g/d for animals fed on cholesterol-free and cholesterol-enriched diets respectively) compared with the other groups $(19 \cdot 5-22 \cdot 3 \mathrm{~g} / \mathrm{d})$. The growth of rats fed on the non-purified diet $(7 \cdot 3(\mathrm{SE} 0 \cdot 1) \mathrm{g} / \mathrm{d})$ was comparable with that of the animals fed on pectin.

Dietary pectin slightly increased the rates of bile flow in rats fed on both cholesterol-free and cholsterol-enriched diets. Pectin, but not cellulose, significantly increased the biliary concentration and excretion of bile acids. Cholesterol supplementation did not modify the effect of dietary fibre on these variables. Bile acid concentration and excretion in rats fed on the non-purified diet were apparently higher than those of rats fed on the various purified diets. In rats fed on the non-purified diet, as demonstrated previously (Ide \& Horii, 1987), the bulk of bile acids was conjugated with taurine. In the groups of rats fed on the cholesterol-free diet containing cellulose or no fibre source compared with those fed on a non-purified diet, biliary concentration of bile acids conjugated with taurine decreased, while those conjugated with glycine remained unchanged. As a consequence, the proportion 


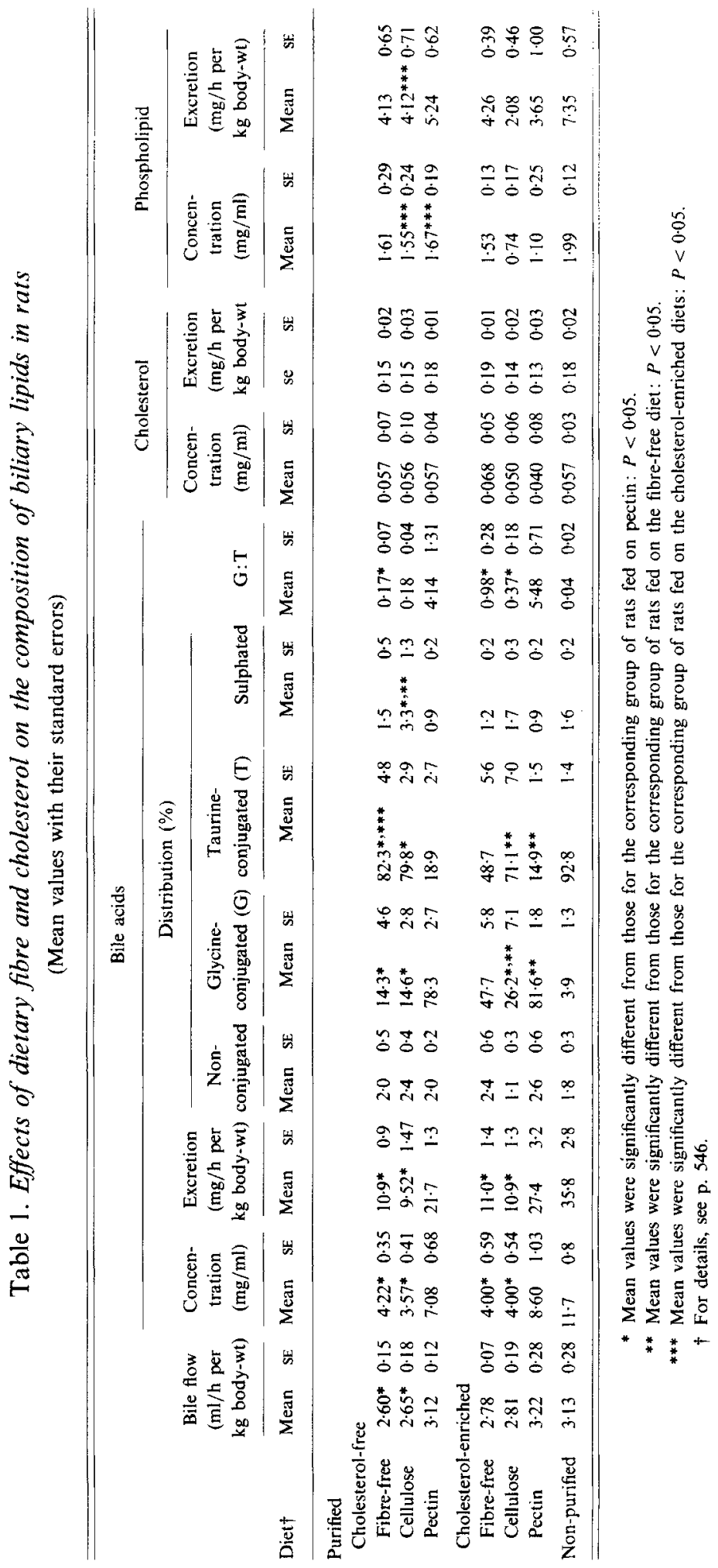


Table 2. Effects of dietary fibre and cholesterol on the faecal steroid excretion of rats (Mean values with their standard errors)

\begin{tabular}{|c|c|c|c|c|c|c|}
\hline \multirow[b]{2}{*}{ Diet ${ }^{\dagger}$} & \multicolumn{2}{|c|}{$\begin{array}{c}\text { Faeces excreted } \\
\text { (g/d per kg body-wt) }\end{array}$} & \multicolumn{2}{|c|}{$\begin{array}{c}\text { Acidic steroids } \\
\text { (mg/d per kg body-wt) }\end{array}$} & \multicolumn{2}{|c|}{$\begin{array}{c}\text { Neutral steroids } \\
\text { (mg/d per kg body-wt) }\end{array}$} \\
\hline & Mean & $\mathrm{SE}$ & Mean & SE & Mean & $\mathbf{S E}$ \\
\hline \multicolumn{7}{|c|}{ Purified } \\
\hline \multicolumn{7}{|c|}{ Cholesterol-free } \\
\hline Fibre-free & $1 \cdot 46^{*}$ & 0.11 & $11 \cdot 5 * * * * *$ & 0.8 & $10 \cdot 7^{* * *}$ & $0 \cdot 9$ \\
\hline Cellulose & $9 \cdot 19^{*, * *}$ & 0.38 & $13 \cdot 0^{*, * * *}$ & $1 \cdot 3$ & $10 \cdot 8^{* * *}$ & 0.6 \\
\hline Pectin & $3 \cdot 19$ & 0.13 & $33 \cdot 8 * * *$ & $2 \cdot 3$ & $21 \cdot 6^{* * *}$ & $1 \cdot 7$ \\
\hline \multicolumn{7}{|c|}{ Cholesterol-enriched } \\
\hline Fibre-free & $1.78^{*}$ & 0.06 & $36 \cdot 4^{*}$ & 2.9 & $178^{*}$ & 7 \\
\hline Cellulose & $9 \cdot 20^{*}, * *$ & 0.31 & 41.6 & $4 \cdot 0$ & $176^{*}$ & 5 \\
\hline Pectin & $3 \cdot 72$ & $0 \cdot 38$ & $43 \cdot 8$ & $2 \cdot 4$ & 240 & 19 \\
\hline Non-purified & $21 \cdot 3$ & 0.4 & $44 \cdot 7$ & $2 \cdot 6$ & $54 \cdot 6$ & $2 \cdot 5$ \\
\hline
\end{tabular}
0.05 .

* Mean valus were significantly different from those for the corresponding group of rats fed on pectin: $P<$

** Mean values were significantly different from those for the corresponding group of rats fed on the fibre-free diet : $P<0.05$.

*** Mean values were significantly different from those for the corresponding group of rats fed on the cholesterol-enriched diets: $P<0.05$.

$\dagger$ For details, see p. 546.

of bile acids conjugated with taurine decreased, but the taurine conjugates still comprised approximately $80 \%$ of biliary bile acids in these rats. Dietary pectin increased the concentration of bile acids conjugated with glycine, while decreasing those conjugated with taurine, in rats fed on both the cholesterol-free and cholesterol-enriched diets. Consequently, the glycine conjugates became predominant in the biliary bile acids of rats fed on pectin. G:T increased to 4-5 in rats fed on pectin, while it remained low (less than 1) in the other groups. Dietary cholesterol also increased the proportion of glycine conjugates, while decreasing that of taurine conjugates. The response was most prominent in rats fed on the fibre-free diet. Non-conjugated and sulphated bile acids comprised only minor proportions with the various dietary treatments. Although types of dietary fibre and cholesterol considerably modified the compositions of bile acids, those of glycine- and taurine-conjugated fractions resembled each other with the various dietary treatments (values not shown).

Dietary fibre and cholesterol did not modify the biliary concentration and excretion of cholesterol. Phospholipid concentration and excretion for the groups of rats fed on cholesterol-free purified diets were similar, while cholesterol feeding decreased the values in rats fed on diets containing cellulose and pectin, but not in those fed on the fibre-free diet.

The dietary fibre, especially cellulose, considerably increased the weight of faceces excreted, while cholesterol feeding did not influence this variable (Table 2). Rats fed on the non-purified diet excreted more faeces than any of the groups of animals fed on the purified diets. Dietary pectin, but not cellulose, increased the excretion of acidic steroids in rats fed on cholesterol-free diets. The cholesterol feeding more than tripled the excretion in rats fed on the fibre-free diet or the diet containing cellulose. On the other hand, the increase due to cholesterol feeding was minimal in rats fed on pectin. As a result, daily bile acid excretions of the three groups of rats fed on the cholesterol-enriched diets were similar, except for a 
slight difference between rats fed on the fibre-free diet and those fed on pectin. Dietary pectin increased neutral steroid excretion in rats fed on both the cholesterol-free and cholesterol-enriched diets; the value for animals fed on the cholesterol-enriched diet was significantly different from those in the other two groups.

\section{Effects of dietary fibre and cholesterol on lumen bile acids}

In the second experiment, lumen bile acids in the groups of rats fed on the purified diets, the compositions of which were the same as those in the previous experiment, were analysed.

Table 3 shows the amounts of bile acids recovered from the lumen of the small intestine and the caecum. As sulphated bile acids were found to comprise negligible proportions of the biliary bile acids (Table 1), these were not determined in this experiment. As in the first experiment, pectin reduced the amount of diet consumed and retarded growth in animals fed on both the cholesterol-free and cholesterol-enriched diets (values not shown). The bile acid pool in the small intestine and caecum of rats fed on pectin increased compared with those of the other treatment groups. Increases in lumen bile acid contents due to pectin feeding were observed in animals fed on both the cholesterol-free and cholesterol-enriched diets. Cholesterol feeding increased ileal but not jejunal bile acid contents in rats fed on cellulose. However, no such effect on jejunal or ileal bile acids could be observed in the animals fed on the fibre-free diet, nor in those fed on pectin. Dietary cholesterol doubled the amount of caecal bile acid in rats fed on the fibre-free diet or the cellulose diet, but not in those fed on pectin.

Considerable proportions of bile acids in the small intestine were found to be deconjugated. The extent of deconjugation was appreciably higher in the ileum than in the jejunum. Cholesterol feeding apparently decreased the proportion of non-conjugated bile acids, especially in rats fed on cellulose. When pooled samples of caecal extracts were analysed, more than $90 \%$ of bile acids were found to be deconjugated in all treatment groups (values not shown). When cholesterol was not included in the diet, the proportions of jejunal and ileal bile acids conjugated with taurine were far greater than those conjugated with glycine, in animals fed on both the fibre-free diet and the cellulose-containing diet. However, G:T was appreciably lower in rats fed on the cellulose-containing diet compared with those fed on the fibre-free diet. As for the biliary bile acids, G:T was increased in rats fed on pectin. Cholesterol feeding increased the proportion of bile acids conjugated with glycine in all groups of rats. Even in this situation, G:T was again lowest in rats fed on cellulose and highest in those fed on pectin.

As in bile, the compositions of the glycine- and taurine-conjugated bile acids in the small intestine resembled each other under the various dietary conditions. On the other hand, those of non-conjugated fractions were completely different from those of these conjugated fractions. The most remarkable differences were observed in cholic and $\beta$-muricholic acids, the predominant primary bile acids in this animal species (Table 4 ). The former was concentrated in the non-conjugated fraction, while the latter was abundant in the conjugated fractions.

Dietary pectin exerts a hypolipidaemic effect in experimental animals as well as in man (Kay, 1982; Kritchevsky, 1987). The lipid-lowering efficacy of pectin was also confirmed in the present study, in animals fed on both the cholesterol-free and cholesterol-enriched diets (Table 5). When cholesterol-free diets were given, serum lipid concentrations in rats fed on the cellulose diet were comparable with those in rats fed on the fibre-free diet, but significantly reduced by pectin feeding. Dietary cholesterol significantly increased the serum cholesterol concentrations in rats fed on the diet free from any fibre source and those fed on cellulose, but not in those fed on pectin. Triglyceride and phospholipid 
Bile acid conjugation in rats

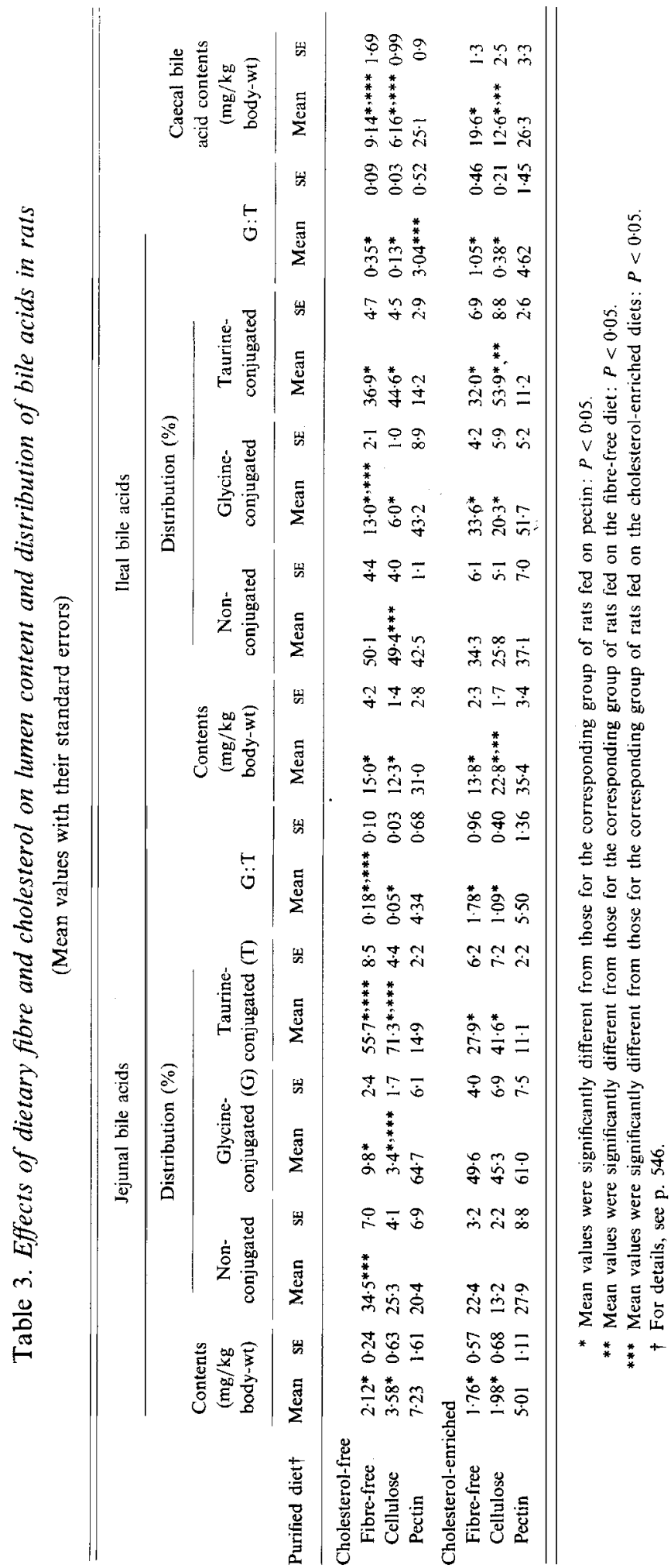




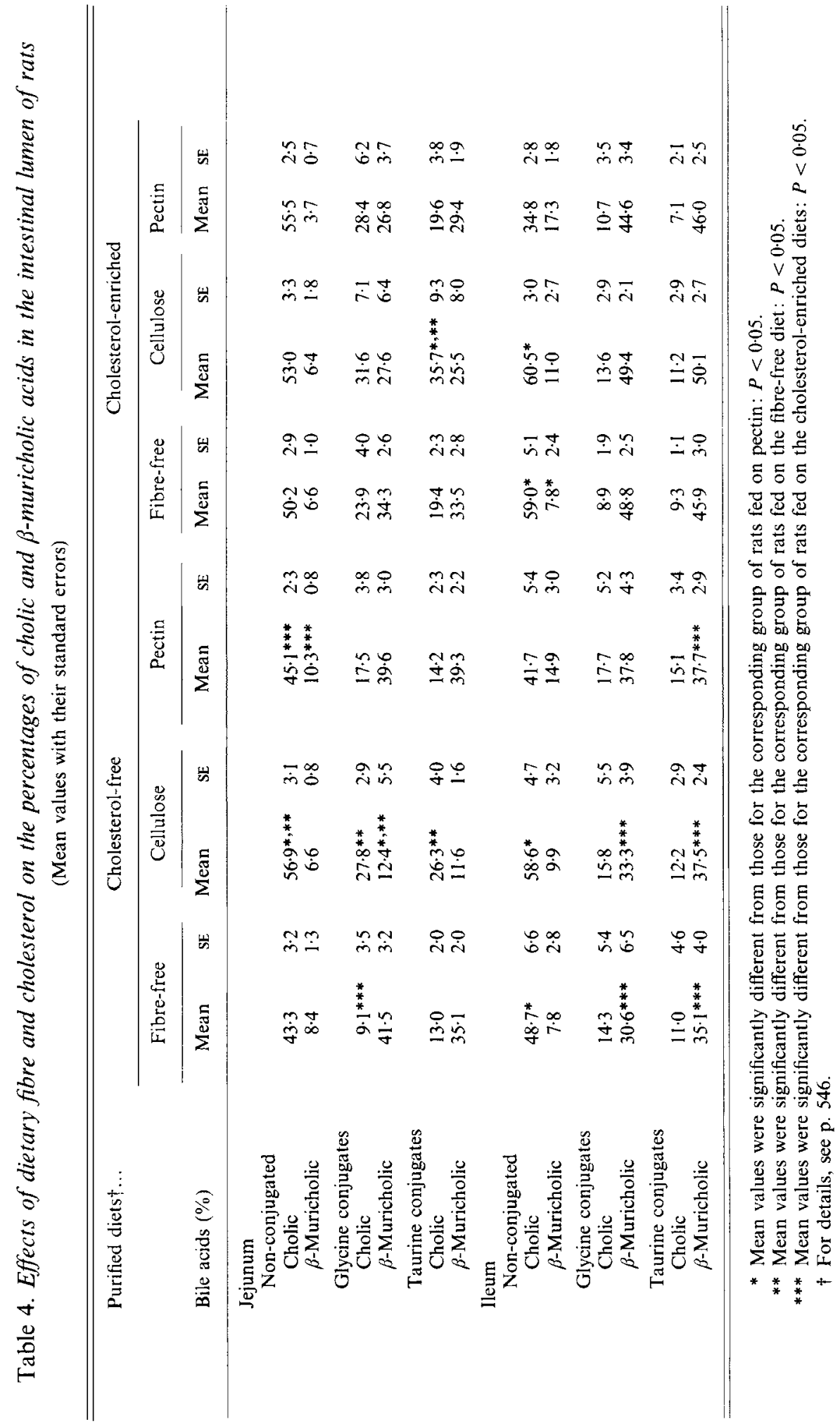


Bile acid conjugation in rats

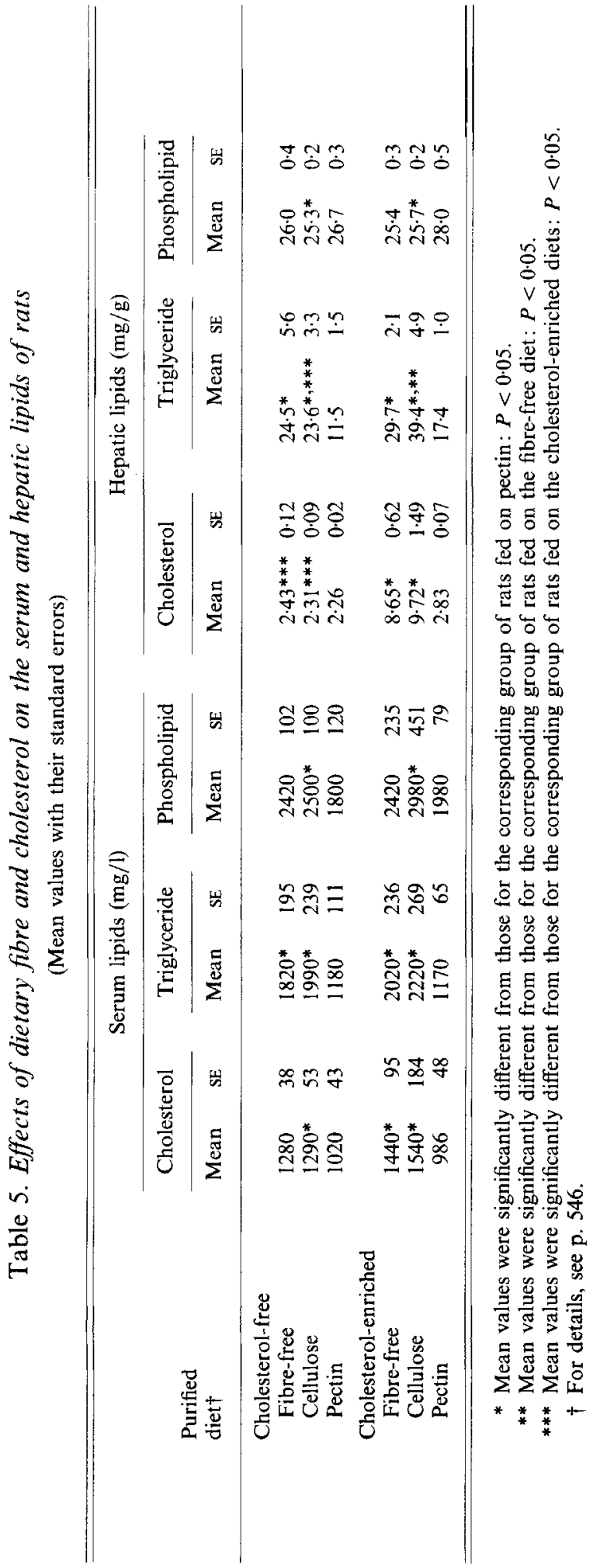


concentrations of serum in rats fed on cholesterol-enriched diets were also lower when pectin was included in the diet compared with the values in rats fed on the fibre-free diet or those in rats fed on cellulose. Hepatic cholesterol concentrations of rats fed on the cholesterol-free diets were comparable. Cholesterol feeding increased the levels of hepatic cholesterol in rats fed on the fibre-free diet and of these fed on cellulose, but only moderately increased the levels in rats, :d on pectin. Dietary pectin also reduced hepatic concentrations of triglyceride, but not of phospholipid, in rats fed on both the cholesterolfree and cholesterol-enriched diets.

\section{DISCUSSION}

The rat has been considered to be the species which conjugates bile acids exclusively with taurine (Haslewood \& Wooton, 1950; Bremer, 1956; Sjövall, 1959; Jacobsen \& Smith, 1968; Spaeth \& Schneider, 1974; Vessey, 1978; Elliott, 1985). However, we have demonstrated a large increase in $\mathrm{G}$ : T in biliary and lumen bile acid, with dietary pectin and cholesterol in this animal species 'Tables 1 and 3). Particularly in rats fed on pectin the glycine conjugates accounted for $8 \mathrm{C} \%$ of biliary bile acids. Increases in the formation of glycine-conjugated bile acids have been demonstrated in rats under particular experimental situations (vitamin $\mathbf{B}_{6}$ and sulphur amino acid deficiencies or partial hepatectomy) where the taurine pool is limited (Bergeret \& Chatagner, 1956; Daisy et al. 1956; Bengmark et al. 1964; Sturman, 1973). Growing evidence indicates that $\mathrm{G}: \mathrm{T}$ in rats is affected by changes in bile acid metabolism (Hardison \& Proffitt, 1977; Yamanaka et al. 1985; Kawamura et al. 1986). The reported findings suggest that the availability of taurine is also a factor which regulates $\mathrm{G}: \mathrm{T}$ under these experimental conditions. As pectin and cholesterol have been shown to alter bile acid metabolism (Dietschy \& Wilson, 1970; Kay, 1982; Kritchevsky, 1987), it is plausible that the modifications in G:T by these dietary factors, as observed in the present work, were also mediated by the alteration of bile acid metabolism and mainly by the availability of taurine. In fact, a preliminary study has demonstrated a significant decrease in the hepatic taurine content in rats fed on a diet containing pectin relative to those fed on a fibre-free diet ( $T$. Ide and $M$. Horii, unpublished results). However, the possibility that an enzyme specific for glycine conjugation may be induced under particular conditions, and thus increase the rate of synthesis of glycineconjugated bile acids relative to that of taurine conjugates should not be disregarded. In case there is only one enzyme specific for taurine in which the $K_{\mathrm{m}}$ value for glycine is 40 times higher than that for taurine (Killenberg \& Jordan, 1978), the hepatic taurine concentration would need to be decreased to levels which are extremely low relative to that of glycine in order to synthesize appreciable amounts of glycine conjugates. However, the situation is not likely to occur in this animal species (Anthony \& Edozien, 1975; Sugiyama et al. 1986). Examination of the hepatic concentrations of these amino acids, as well as the substrate specificity of the bile acid conjugation enzyme, in rats fed on cholesterol or pectin would be required in order to draw a definite conclusion.

Though various factors modify the relative conjugation of bile acids with glycine and taurine in man (Sjövall, 1959; Lindstedt et al. 1965; Truswell et al. 1965; Garbutt et al. 1971; Garbutt \& Kenney, 1972; Hardison, 1978; Stephan et al. 1981), the physiological significance is little understood. Bile salts appear to be required for cholesterol but not triglyceride absorption (Borgström et al. 1985; Stange \& Dietschy, 1985). Thus, it is plausible that the absorption and metabolism of cholesterol rather than of triglyceride is profoundly modified by the alteration of the distribution of bile acids between glycine and taurine conjugates. Available evidence (Portman \& Man, 1955; Sjövall, 1959; Hermann, 1959; Howe et al. 1960; Bengmark et al. 1964; Truswell et al. 1965; Dietschy \& Wilson, 
1970; Tamesue et al. 1973; Stephan et al. 1981 ; Yamanaka et al. 1986; Armstrong \& Carey, 1987) suggests that cholesterol solubilization and absorption are stimulated more in the presence of glycine-conjugated bile acids than in the presence of taurine conjugates. If this is the case, it could be speculated that the increase in the formation of glycine conjugates in rats fed on pectin was the adaptive response of the animals which stimulates cholesterol solubilization and absorption under conditions where the absorptive process is seriously hindered (Table 2) (Kay, 1982; Kritchevsky, 1987). The dietary cholesterol-mediated increase in $\mathrm{G}: T$ could also be regarded as the adaptive response of the animals which is necessary to solubilize a large amount of incoming cholesterol in the intestinal lumen. Detailed studies would be required to test this hypothesis.

Some types of dietary fibre have been shown to increase the lumen bile acid contents (Eastwood \& Boyd, 1967; Boyd \& Eastwood, 1968). In the present study, lumen bile acid contents, in both the small intestine and caecum, were markedly increased by dietary pectin but not by cellulose (Table 3). Eastwood \& Boyd (1967) and Boyd \& Eastwood (1968) suggested that the capacity of dietary fibre to adsorb bile salts was responsible for the changes in lumen bile acid. However, no definite relation between the apparent bile acid adsorption capacities of dietary fibre (Story \& Lord, 1987) and the lumen bile acid contents (Eastwood \& Boyd, 1967; Boyd \& Eastwood, 1968; Schneeman \& Gallaher, 1980; Sheard \& Schneeman, 1980) appears to exist. As dietary pectin, but not cellulose, was found to increase the biliary bile acid concentration and excretion in the present study, the increased influx of bile acids into the lumen, rather than the differences in the bile acid absorption capacities between these dietary fibres, may be responsible for the change in the lumen bile acid pool observed here. In this context rats fed on the non-purified diet, compared with those fed on the various purified diets, excreted more bile acid into the bile (Table 1). This is compatible with the observation that the lumen bile acid pool in rats fed on the nonpurified diet is considerably greater than that of animals fed on the purified diet (Fisher et al. 1975; Ide \& Horii, 1987).

Bacterial hydrolysis of the peptide bond of conjugated bile acids has been considered to be mainly accomplished in the large intestine (Macdonald et al. 1983; Hylemon, 1985). In the present study, however, a considerable proportion of lumen bile acids was found to be deconjugated even in the upper part of the small intestine (Table 4); this confirmed the results of a previous study in rats fed on a non-purified diet (Ide \& Horii, 1987). Sakai \& Kawai (1981) have found that conjugated bile acids merely represented $2 \%$ of total bile acids in the ileum of rats fed on a non-purified diet. The dietary cholesterol and various types of bile acids increased the proportion of conjugated bile acids to varying extents $(28-56 \%)$. Although further information regarding the extent of bile acid deconjugation in the lumen of the small intestine has hitherto been lacking, these results, at least, indicate that bacterial deconjugation of bile acids occurs to a considerable event even in the small intestine. The abundance of cholic acid in the non-conjugated fraction (Table 4) indicates that the bacterial strain(s) which specifically deconjugate cholic acid (Chikai et al. 1987) is prominent in the small intestine under the present conditions.

In conclusion, the relative amouns of glycine- and taurine-conjugated bile acids in the bile and lumen of the small intestine could be profoundly modified by dietary manipulations in rats. Although rats have been regarded as a species which conjugates bile acids almost exclusively with taurine (Haselwood \& Wooton, 1950; Bremer, 1956; Jacobsen \& Smith, 1968; Killenberg, 1978; Elliott, 1985), except for several observations to the contrary (Bergeret \& Chatagner, 1956; Daisy et al. 1956; Bengmark et al. 1964; Yamanaka et al. 1985; Kawamura et al. 1986), the predominant pro-portions of both biliary and lumen bile acids in the present study were conjugated with glycine in the animals fed on pectin. The diet-induced alterations in the partition of bile acids between glycine and taurine may also 
have a physiological significance in the regulation of bile acid and lipid metabolism in this animal species.

The authors wish to thank Yasuyuki Komori and Akiko Okayama for their excellent technical assistance.

\section{REFERENCES}

American Institute of Nutrition (1977). Report of the American Institute of Nutrition ad hoc committee on standards for nutritional studies. Journal of Nutrition 107, 1340-1348.

Anthony, L. \& Edozien, J. C. (1975). Experimental protein and energy deficiencies in the rat. Journal of Nutrition $105,631-648$

Armstrong, M. J. \& Carey, M. C. (1987). Thermodynamic and molecular determinants of sterol solubilities in bile salt micelles. Journal of Lipid Research 28, 1144-1155.

Bengmark, S., Ekdahl, P.-H. \& Olsson, R. (1964). Effect of taurine and glycinc treatment on the conjugation of bile acids in partially hepatectomized rats. Acta Chirurgica Scandinavica 128, 180-185.

Bergeret, B. \& Chatagner, F. (1956). Influence d'une carence en vitamin $\mathbf{B}_{6}$ sur la teneur en acids tauro-conjugatés et glyco-conjugatés de la bile du rat. Biochimica et Biophysica Acta 22, 273-277.

Borgström, B., Barrowman, J. A. \& Lindström, M. (1985). Role of bile acids in intestinal lipid digestion and absorption. In Sterols and Bile Acids, pp. 405-425 [H. Danielsson and J. Sjövall, editors]. Amsterdam : Elsevier.

Boyd, G. S. \& Eastwood, M. A. (1968). Studies on the quantitative distribution of bile salts along the rat small intestine under varying dietary regimens. Biochimica et Biophysica Acta 152, 159-164.

Bremer, J. (1956). Species differences in the conjugation of free bile acids with taurine and glycine. Biochemical Journal 63, 507-513.

Chikai, T., Nakao, H. \& Uchida, K. (1987). Deconjugation of bile acids by human intestinal bacteria implanted in germ-free rats. Lipids 22, 669-671.

Daisy, E. A. Jr, Daniels, M. \& Zimmerman, M. A. (1956). Nutritional influences on conjugation of bile acids by the rat. Federation Proceedings 15, 243-248.

Dietschy, J. M. \& Wilson, J. D. (1970). Regulation of cholesterol metabolism. New England Journal of Medicine 282, 1128-1136, 1179-1183, 1241-1249.

Eastwood, M. A. \& Boyd, G. S. (1967). The distribution of bile salts along the small intestine of rats. Biochimica et Biophysica Acta 137, 393-396.

Elliott, W. E. (1985). Metabolism of bile acid in liver and extrahepatic tissues. In Sterols and Bile Acids, pp. 303-329 [H. Danielsson and J. Sjövall, editors]. Amsterdam: Elsevier.

Fisher, M. M., Kakis, G. \& Yousef, I. M. (1975). Bile acid pool in Wistar rats. Lipids 11, 93-96.

Folch, J., Lee, M. \& Sloane-Stanley, G. H. (1957). A simple method for the isolation and purification of total lipids from animal tissues. Journal of Biological Chemistry 226, 497-509.

Garbutt, J. T. \& Kenney, T. J. (1972). Effect of cholestyramine on bile acid metabolism in normal man. Journal of Clinical Investigation 51, 2781-2789.

Garbutt, J. T., Lack, L. \& Tyor, M. P. (1971). Physiological basis of alterations in the relative conjugation of bile acids with glycine and taurine. American Journal of Clinical Nutrition 24, 218-228.

Goto, J., Kato, H., Sawata, Y. \& Nambara, T. (1981). Separation and determination of bile acid 3-sulphates in human bile by high-performance liquid chromatography. Journal of Chromatography 226, 13-24.

Hardison, W. G. M. (1978). Hepatic taurine concentration and dietary taurine as regulators of bile acid conjugation with taurine. Gastroenterology 75, 71-75.

Hardison, W. G. M. \& Proffitt, J. H. (1977). Influence of hepatic taurine concentration on bile acid conjugation with taurine. American Journal of Physiology 232, E75-E79.

Haslewood, G. A. D. \& Wooton, V. (1950). Comparative studies of bile salts. 1. Preliminary survey. Biochemical Journal 47, 584-597.

Hermann, R. G. (1959). Effect of taurine, glycine and $\beta$-sitosterol on serum and tissue cholesterol in the rat and rabbit. Circulation Research 7, 224-227.

Howe, E. E., Bosshardt, D. K. \& Huff, J. W. (1960). Effect of bile acids on plasma cholesterol in the mouse. Journal of Nutrition 72, 379-386.

Hylemon, P. B. (1985). Metabolism of bile acids in intestinal microflora. In Sterols and Bile Acids, pp. 331-343 [H. Danielsson and J. Sjövall, editors]. Amsterdam: Elsevier.

Ide, T. \& Horii, M. (1987). A simple method for the extraction and determination of non-conjugated and conjugated luminal bile acids in rats. Agricultural and Biological Chemistry 51, 3155- 3157.

Ide, T., Okamatsu, H. \& Sugano, M. (1978). Regulation by dietary fats of 3-hydroxy-3-methylglutaryl-Coenzyme A reductase in rat liver. Journal of Nutrition 108, 601-612.

Ide, T., Oku, H. \& Sugano, M. (1982). Reciprocal responses to clofibrate in ketogenesis and triglyceride and cholesterol secretion in isolated rat liver. Metabolism 31, 1065-0172.

Jacobsen, J. G. \& Smith, L. H. Jr (1968). Biochemistry and physiology of taurine derivatives. Physiological Reviews 48, 424-511. 
Kawamura, M.. Tsuji, K., Nakagawa, Y. \& Ichikawa, T. (1986). Suppression of hypercholesterolemia by glutathione feeding in rats. Sulfur Amino Acids $9,327 \cdots 332$.

Kay, R. M. (1982). Dietary fiber. Journal of Lipid Research 23, 221242.

Killenberg, P. G. (1978). Measurement and subcellular distribution of choloyl-CoA: amino acid $N$ acyltransferase activities in rat liver. Journal of Lipid Research 19, 24-31.

Killenberg, P. G. \& Jordan, J. T. (1978). Purification and characterization of bile acid-CoA: amino acid $N$ acyltransferase from rat liver. Journal of Biological Chemistry 235, 10051010.

Kritchevsky, D. (1987). Dietary fiber and lipid metabolism in animals. Scandinavian Joumal of Gastronterolog." 22, Suppl. 129, 213217.

Kuriyama, K., Ban, Y.\& Nakashima, T. (1979). Simultaneous determination of biliary bile acids in rat: electron impact and ammonia chemical ionization mass spectrometric analyses of hile acids. Steroids 34, 717-728.

Lindstedt, S., Avigan, J., Goodman, De D. W., Sjövall, J. \& Steinberg, D. (1965). The effect of dietary fat on the turnover of cholic acid and on the composition of biliary bile acids in man. Journal of Clinical Investigation 44. $1754-1765$.

Macdonald, I. A., Bokkenheuser, V. D., Winter, J., McLernon, A. M. \& Mosbach, E. H. (1983), Degradation of steroids in the human gut. Journal of Lipid Research 24, 675 700.

Portman, O. W. \& Man, G. V. (1955). The disposition of taurine-S $S^{35}$ and taurocholate- $S^{35}$ in the rat: dietary influences. Journal of Biological Chemistry 213, 733-743.

Sakai, K. \& Kawai, Y. (1981). Potential activity of bile acids in carcinogenicity. In Intestinal Flora and Carcinogenesis, pp. 41-62 [T. Mitsuoka, editor]. Tokyo: Gakkai Syuppan Center.

Schneeman, B. O. \& Gallaher. D. (1980). Changes in small intestinal digestive enzyme activity and bilc acids with dietary cellulose in rats. Journal of Nutrition $110,584-590$.

Sheard, N. F. \& Schneeman, B. O. (1980). Wheat bran's effect on digestive enzyme activity and bile acid levels in rats. Journal of Food Science 45, 1645-1648.

Sjövall, J. (1959). Dietary glycine and taurine on bile acid conjugation in man. Proceedings of the Society for Experimental Biology and Medicine 100, 676-678.

Snedecor, G. W. \& Cochran, W. G. (1967). Statistical Methods, 6th ed. Ames, IA: Iowa State University Press.

Spaeth, D. G. \& Schneider, D. L. (1974). Taurine synthesis, concentration, and bile salt conjugation in rat, guineapig, and rabbit. Proceedings of the Society for Experimental Biology and Medicine 147, 855-858.

Stange, E. F. \& Dietschy, J. M. (1985). Cholesterol absorption and metabolism by the intestinal cpithelium. In Sterols and Bile Acids, pp. 121-149 [H. Danielsson and J. Sjövall, editors]. Amsterdam: Elsevier.

Stephan, Z. F., Armstrong, M. J. \& Hayes, K. C. (1981). Bile acid alterations in taurine-depleted monkeys. American Journal of Clinical Nutrition 34, 204-210.

Story, J. A. \& Lord, S. L. (1987). Bile salts: in vivo studies with fiber components. Scandinavian Journal of Gastroenterology 22, Suppl. 129, 174 180.

Sturman, J. A. (1973). Taurine pool size in the rat: effects of vitamin B-6 deficiency and high taurine diet. Journal of Nutrition 103, 1566-1580.

Sugano, M., Ide, T., Kohno, M., Watanabe, M., Cho, Y.-J. \& Nagata, Y. (1983). Biliary and fecal steroid excretion in rats fed partially hydrogenated soybean oil. Lipids 18, 186-192.

Sugiyama, K., Akai, H. \& Muramatsu, K. (1986). Journal of Nutritional Science and Vitaminolog, $32,537549$.

Tamesue, N., Inoue, T.\& Juniper, K. (1973). Solubility of eholesterol in bile salt lecithin model systems. Digestive Disease 18, 670-678.

Truswell, A. S., McVeigh, S., Mitchell, W. D. \& Bronte-Stewart, B. (1965). Effect in man of feeding taurine on bile acid conjugation and serum cholesterol levels. Journal of Atherosclerosis Research 5, 523-526.

Uchida, K., Nomura, Y., Kadowaki, M., Takeuchi, N. \& Yamamura, Y. (1977). Effect of dietary cholesterol on cholesterol and bile acid metabolism in rats. Japanese Journal of Pharmacology 27, 193-204.

Vessey, D. A. (1978). The biochemical basis for the conjugation of bile acid with either glycine or taurine. Biochemical Journal 174, 621-626.

Yamanaka, Y., Tsuji, K. \& Ichihara, T. (1986). Stimulation of chenodeoxycholic acid excretion in hypercholesterolemic mice by dietary taurine. Journal of Nutritional Science and Vitaminology 32, 287. 296.

Yamanaka, Y., Tsuji, K., Ichikawa, T., Nakagawa, Y. \& Kawamura, M. (1985). Effect of dietary taurine and taurocyamine on biliary glycine/taurine ratio and cholesterol and bile acid metabolisms. Sulfur Amino Acids 8 , $209-214$. 